\title{
Comparison of Two Different Fibrinogen Concentrates in an in vitro Model of Dilutional Coagulopathy
}

\author{
Philipp Groene $^{a}$ Tobias Wiederkehr ${ }^{a}$ Tobias Kammerer ${ }^{a}$ b Patrick Möhnle ${ }^{a}$ \\ Melanie Maerte $^{\mathrm{a}} \quad$ Andreas Bayer $^{\mathrm{a}} \quad$ Klaus Görlinger $^{c} \quad$ Markus Rehm $^{\mathrm{a}}$ \\ Simon T. Schäfer ${ }^{a}$ \\ ${ }^{a}$ Department of Anaesthesiology, University Hospital, LMU Munich, Munich, Germany; ${ }^{\mathrm{b}}$ Institute for \\ Anaesthesiology and Pain Therapy, HDZ NRW, Bad Oeynhausen, Germany; ${ }^{C}$ Medical Director, TEM Innovations, \\ Munich, Germany
}

\author{
Keywords \\ Coagulation disorders · Blood coagulation tests · Colloids · \\ Fibrinogen · Thromboelastometry
}

\begin{abstract}
Introduction: Fibrinogen concentrates are widely used to restore clot stability in situations of bleeding. Fibrinogen preparations are produced using different production methods, resulting in different compounds. Thus, different preparations might have a distinct impact on blood coagulation. We tested the effect of fibrinogen concentrates Haemocomplettan ${ }^{\circledR}$ (CSL Behring, Marburg, Germany) and fibryga ${ }^{\circledR}$ (Octapharma $\mathrm{GmbH}$, Langenfeld, Germany) on the impairments induced by $60 \%$ dilutional coagulopathy in vitro. Materials and Methods: The influence of the fibrinogen concentrates fibryga ${ }^{\circledR}$ and Haemocomplettan ${ }^{\circledR}$ on colloid (gelatine, hydroxyethyl starch [HES], albumin)-induced or crystalloid (Ringer's acetate)-induced dilutional coagulopathy was analysed using rotational thromboelastometry $\left(\right.$ ROTEM $\left.^{\circledR}\right)$ and standard laboratory tests. The following experimental conditions were analysed in vitro: whole blood, $60 \%$ dilution ( $40 \%$ blood and $60 \%$ diluent) \pm 50 or $100 \mathrm{mg} /$ $\mathrm{kg}^{-1}$ fibryga ${ }^{\circledR}$ or Haemocomplettan ${ }^{\circledR}$, respectively. Results: Dilution with either diluent resulted in prolonged clotting time $(C T)$ in an extrinsic activated test $\left(\mathrm{CT}_{\text {EXTEM }}\right)$ and decreased maximum clot firmness $\left(\mathrm{MCF}_{\mathrm{FIBTEM}}\right)$ as expressed, e.g., by gelatine: (59.5 s [62/54.8] vs. 95 s [102.8/86.8]; $p<$
\end{abstract}

0.001 and $14 \mathrm{~mm}[16 / 10.5]$ vs. $3 \mathrm{~mm}$ [4-3]; $p<0.001)$. Substitution after $60 \%$ dilution with HES resulted in no difference between the preparations, except for shorter thrombin time with fibryga ${ }^{\circledR}$ (14 s [15/14] vs. 18 s [18.8/17]; $p=0.0093$; low dose). $\mathrm{CT}_{\text {EXTEM }}$ was higher with Haemocomplettan ${ }^{\circledR}$ in a gelatine-induced dilution (51 s [54.5/47.5] vs. $63 \mathrm{~s}$ [71/60.3]; $p=$ 0.0202 ; low dose) whereas thrombin time was lower with fibryga $^{\circledR}(19.5$ s [20.8/19] vs. 27 s [29/25.3]; $p=0.0017)$. In dilution with albumin, differences in $\mathrm{CT}_{\text {EXTEM }}(69 \mathrm{~s}$ [76.5/66] vs. $56 \mathrm{~s}$ [57/53.3]; $p=0.0114$; low dose) and thrombin time (18 s [18/17] vs. 24.5 s [25.8/24]; $p=0.0202$; low dose) were seen. In dilution with crystalloid solution, again differences in $\mathrm{CT}_{\text {EXTEM }}$ (53.5 s [57.8/53] vs. $45 \mathrm{~s}$ [47/43]; $p=0.035$; low dose) and thrombin time (17 s [17/16] vs. 23.5 s [24/23]; $p=0.0014$; low dose) were seen. Fibrinogen levels were more increased by high-dose substitution of both preparations. Conclusion: Based on this data it can be stated that both fibryga ${ }^{\circledR}$ and Haemocomplettan ${ }^{\circledR}$ had the same performance in our in vitro model except for $\mathrm{CT}_{\text {EXTEM }}$ and thrombin time.

๑) 2019 S. Karger AG, Basel

\section{Introduction}

Severe haemorrhage results in reduced blood volume as well as loss of erythrocytes and coagulation factors. The loss of blood volume is primarily treated with infusion solutions (e.g., crystalloids and colloids) or packed red 
cells. Furthermore, dilutional coagulopathy is composed of both, consumption and loss of coagulation factors, which is substituted using fresh frozen plasma and coagulation factor preparations. In this regard, fibrinogen is of particular interest as it is one of the first coagulation factors reaching critical low plasma concentrations during bleeding [1-3]. Fibrinogen concentrate substitution, traditionally administered in hereditary hypofibrinogenemia, is also becoming more common as an early treatment option in acquired deficiency.

Previous studies have shown the in vitro and in vivo effects of crystalloid and colloid infusion on thromboelastometry (e.g., ROTEM ${ }^{\circledR}$ )-based blood coagulation measurements, focussing on maximum clot firmness

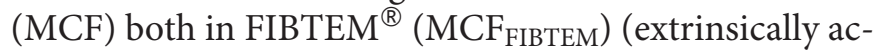
tivated viscoelastic assay with blocked platelet function to evaluate fibrinogen-dependent clot stability) and EX$\mathrm{TEM}^{\circledR}\left(\mathrm{MCF}_{\text {EXTEM}}\right)$ (extrinsically activated viscoelastic assay to evaluate the extrinsic coagulation pathway) tests and clotting time (CT) in EXTEM $^{\circledR}\left(\mathrm{CT}_{\text {EXTEM }}\right)$ [4-9]. Furthermore, it has been shown that fibrinogen administration can partially reverse dilutional coagulopathy [46]. Therefore, we chose a similar way in an in vitro model of dilutional coagulopathy to test the performance of a new fibrinogen concentrate compared to an established product.

Clinicians might emphasize that one fibrinogen preparation equals another. However, there are differences in production techniques, quality controls, as well as rules and regulations. In detail, it is not the actual amount of fibrinogen that is measured and added to every single vial; in contrast, total protein including a variety of further coagulation factors is used. Thus, different preparations can differ by $20 \%$ regarding fibrinogen and even more for other clotting proteins. Thus, there can be relevant differences between 2 distinct preparations of fibrinogen. This might impact on the effect in dilutional coagulopathy, that is, following administration of crystalloids or colloids, and has been investigated in this study.

We tested the hypothesis that the new fibrinogen concentrate fibryga ${ }^{\circledR}$ (Octapharma GmbH, Langenfeld, Germany) is equally effective in reversing an in vitro $60 \%$ dilutional coagulopathy induced by a crystalloid and various colloids compared to the established fibrinogen concentrate Haemocomplettan ${ }^{\circledR}$ P (CSL Behring, Marburg, Germany).

\section{Materials and Methods}

Healthy volunteers were recruited by bulletins at our institution and enrolled after written informed consent. Twelve healthy individuals, without coagulation disorders or intake of anticoagulants, were included in this prospective trial and volunteer characteristics were documented. Venous blood samples (S-Monovette ${ }^{\circledR}$
Table 1. Characteristics of healthy volunteers

Gender (male/female), $n$ (\%)

Age, years

Body mass index, $\mathrm{kg} \mathrm{m}^{-2}$

$6 / 6(50 / 50)$

$38.3 \pm 8.9$

$23.7 \pm 3.0$

$0.94 \pm 0.09$

Creatinine serum concentration, $\mathrm{mg} / \mathrm{dL}$

$4.5 \pm 0.3$

$4.9 \pm 0.5$

$14.4 \pm 1.3$

$0.43 \pm 0.04$

$5.2 \pm 0.6$

$243 \pm 45$

$1(1-1)$

$22.7 \pm 2.8$

$277 \pm 62$

$17.9 \pm 1.4$

$4 / 8(33 / 67)$

Thrombin time, $s$

Medication intake (yes/no), $n(\%) *$

Values are mean \pm SD unless otherwise indicated. INR, international normalized ratio; PTT, partial thromboplastin time. * Medication intake: pantoprazol $(n=1)$, L-thyroxin $(n=1)$, Thyronajod $(n=1)$, contraceptive pill $(n=1)$.

Citrate 3.2\%; Sarstedt, Nümbrecht, Germany) were withdrawn twice within $4 \mathrm{~h}$ and processed within $2 \mathrm{~h}$.

Blood coagulation was assessed by both thromboelastometry $\left(\right.$ ROTEM $\left.^{\circledR}\right)$ and standard laboratory tests at baseline (undiluted citrated blood), following a 60\% dilution with colloids (hydroxyethyl starch [HES], gelatine, or albumin) or a balanced crystalloid solution ( $60 \%$ diluent $+40 \%$ blood), and following dilution plus either of 2 fibrinogen dosages of both concentrates (80 and 160 $\left.\mathrm{mg} / \mathrm{dL}^{-1}\right)$.

\section{Sample Preparation}

The influence of 3 colloids - HES 6\% 130/0.4 (Voluven ${ }^{\circledR}$; Fresenius Kabi Deutschland GmbH, Bad Homburg, Germany), succinylated gelatine $4 \%$ (Gelafundin ${ }^{\circledR} 4 \%$; Braun Melsungen AG, Melsungen, Germany), and human serum albumin (Albunorm ${ }^{\circledR} 5 \%$; Octapharma $\mathrm{GmbH}$ ) and balanced Ringer's acetate (Jonosteril ${ }^{\circledR}$; Fresenius Kabi Deutschland $\mathrm{GmbH}$ ) - on blood coagulation (with and without fibrinogen substitution) was assessed. Diluents were added to citrated tubes prior to all experiments to equalize citrate concentrations in blood and dilutional agents. Dilutions were done by mixing $680 \mu \mathrm{L}$ of citrated whole blood and 1,020 $\mu \mathrm{L}$ of citrated diluent in $2-\mathrm{mL}$ micro tubes $\left(\right.$ Sarsted $\left.{ }^{\circledR}\right)$. fibryga ${ }^{\circledR}$ (Octapharma GmbH) and Haemocomplet$\tan ^{\circledR} \mathrm{P}$ (CSL Behring) were dissolved in water for injection (Aqua ad iniectabilia; Berlin Chemie AG, Berlin, Germany) to a final concentration of $20 \mathrm{mg} / \mathrm{mL}^{-1}$ based on the labelled quantity of $1 \mathrm{~g}$ per vial. A new 1 -g fibrinogen concentrate vial from the same production lot was used every day. Fibrinogen was filtered following the manufacturer's instructions using a $17-\mu \mathrm{m}$ filter (PALL Medical ${ }^{\circledR}$, Port Washington, USA) to ensure particle free solutions.

Six different experimental conditions were analysed per diluent ( 24 conditions in total): undiluted blood, $60 \%$ dilution ( $40 \%$ blood and $60 \%$ diluent) \pm low-dose and high-dose fibryga ${ }^{\circledR}$ or Haemocomplettan ${ }^{\circledR}$ P. For low-dose fibrinogen substitution, $68 \mu \mathrm{L}(1.36$ $\mathrm{mg}$ ) of fibrinogen concentrate was added to $1,700 \mu \mathrm{L}$ of citrated blood $\left(0.8 \mathrm{mg} / \mathrm{mL}^{-1}\right.$ or $\left.80 \mathrm{mg} / \mathrm{dL}^{-1}\right)$. This corresponds to a clinical dose of $50 \mathrm{mg} / \mathrm{kg}^{-1}$ (or $4 \mathrm{~g}$ per $80 \mathrm{~kg}$ body weight). For high-dose 
Table 2. Dilution with hydroxyethyl starch

\begin{tabular}{|c|c|c|c|c|c|c|c|c|c|c|c|}
\hline $\begin{array}{l}\text { Hydroxyethyl } \\
\text { starch }\end{array}$ & $\begin{array}{l}\text { Undiluted } \\
\text { blood }\end{array}$ & $\begin{array}{l}60 \% \\
\text { dilution }\end{array}$ & $p$ & $\begin{array}{l}\text { fibryga } \\
\text { low dose }\end{array}$ & $p$ & $\begin{array}{l}\text { Haemo- } \\
\text { complettan } \\
\text { low dose }\end{array}$ & $p$ & $\begin{array}{l}\text { fibryga } \\
\text { high dose }\end{array}$ & $p$ & $\begin{array}{l}\text { Haemo- } \\
\text { complettan } \\
\text { high dose }\end{array}$ & $p$ \\
\hline $\mathrm{CT}_{\text {EXTEM }}, \mathrm{s}$ & $\begin{array}{l}59.5 \\
(58.25 / 65.25)\end{array}$ & $\begin{array}{l}154 \\
(134.8 / 166)\end{array}$ & $<0.0001^{*}$ & $\begin{array}{l}100 \\
(78.5 / 118.3)\end{array}$ & $\begin{array}{l}0.0293^{*} \\
0.0592^{\#}\end{array}$ & $\begin{array}{l}137 \\
(113.8 / 143.5)\end{array}$ & $\begin{array}{r}0.0001^{*} \\
>0.9999^{\#}\end{array}$ & $\begin{array}{l}69.5 \\
(60.3 / 76.8)\end{array}$ & $\begin{array}{l}>0.9999^{*} \\
<0.0001^{\#}\end{array}$ & $\begin{array}{l}76.5 \\
(57 / 91.3)\end{array}$ & $\begin{array}{l}>0.9999^{*} \\
<0.0001^{\#}\end{array}$ \\
\hline $\mathrm{MCF}_{\text {EXTEM}}, \mathrm{mm}$ & $\begin{array}{l}59 \\
(57 / 65.75)\end{array}$ & $\begin{array}{l}31 \\
(28.3 / 37.8)\end{array}$ & $<0.0001^{*}$ & $\begin{array}{l}39 \\
(37 / 43.8)\end{array}$ & $\begin{array}{l}0.0017^{*} \\
0.1148^{\#}\end{array}$ & $\begin{array}{l}38 \\
(33.75 / 42)\end{array}$ & $\begin{array}{l}<0.0001^{*} \\
>0.9999^{\#}\end{array}$ & $\begin{array}{l}46.5 \\
(43 / 48.8)\end{array}$ & $\begin{array}{l}>0.9999^{*} \\
<0.0001^{\#}\end{array}$ & $\begin{array}{l}42 \\
(37.3 / 44.8)\end{array}$ & $\begin{array}{l}0.0419^{*} \\
0.0062^{\#}\end{array}$ \\
\hline $\mathrm{MCF}_{\text {FIBTEM }}, \mathrm{mm}$ & $\begin{array}{l}13 \\
(11 / 18.5)\end{array}$ & $\begin{array}{l}0 \\
(0 / 0)\end{array}$ & $<0.0001^{*}$ & $\begin{array}{l}3 \\
(3 / 4)\end{array}$ & $\begin{array}{l}0.0009^{*} \\
0.3289^{\#}\end{array}$ & $\begin{array}{l}2.5 \\
(0 / 3.75)\end{array}$ & $\begin{array}{l}<0.0001^{*} \\
>0.9999^{\#}\end{array}$ & $\begin{array}{l}6 \\
(5 / 6.75)\end{array}$ & $\begin{array}{l}>0.9999^{*} \\
<0.0001^{\#}\end{array}$ & $\begin{array}{l}4 \\
(4 / 5)\end{array}$ & $\begin{array}{l}0.0592^{*} \\
0.0093^{\#}\end{array}$ \\
\hline $\begin{array}{l}\text { Fibrinogen } \\
\text { Plasma, } \\
\mathrm{mg} / \mathrm{dL}\end{array}$ & $\begin{array}{l}253.5 \\
(226.3 / 322.3)\end{array}$ & $\begin{array}{l}121 \\
(95.5 / 17.8)\end{array}$ & $0.0017^{*}$ & $\begin{array}{l}219.5 \\
(201.5 / 237)\end{array}$ & $\begin{array}{r}0.4962^{*} \\
>0.9999^{\#}\end{array}$ & $\begin{array}{l}225.5 \\
(208.5 / 237)\end{array}$ & $\begin{array}{r}>0.9999^{*} \\
0.2853^{\#}\end{array}$ & $\begin{array}{l}408 \\
(373 / 430.8)\end{array}$ & $\begin{array}{l}>0.9999^{*} \\
<0.0001^{\#}\end{array}$ & $\begin{array}{l}425.5 \\
(390.8 / 4.413)\end{array}$ & $\begin{array}{r}0.2129^{*} \\
<0.0001^{\#}\end{array}$ \\
\hline aPTT, s & $\begin{array}{l}28 \\
(26.25 / 29.75)\end{array}$ & $\begin{array}{l}78.5 \\
(67 / 91.8)\end{array}$ & $<0.0001^{*}$ & $\begin{array}{l}74 \\
(63.3 / 85.8)\end{array}$ & $\begin{array}{l}0.0062^{*} \\
0.1832^{\#}\end{array}$ & $\begin{array}{l}73.5 \\
(64 / 84.5)\end{array}$ & $\begin{array}{l}0.0167^{*} \\
0.0829^{\#}\end{array}$ & $\begin{array}{l}73.5 \\
(60.3 / 84.3)\end{array}$ & $\begin{array}{l}0.0202^{*} \\
0.0702^{\#}\end{array}$ & $\begin{array}{l}73 \\
(64.3 / 85.8)\end{array}$ & $\begin{array}{l}0.0017^{*} \\
0.4338^{\#}\end{array}$ \\
\hline INR & $\begin{array}{l}1 \\
(1 / 1)\end{array}$ & $\begin{array}{l}1.9 \\
(1.8 / 2.1)\end{array}$ & $<0.0001^{*}$ & $\begin{array}{l}1.75 \\
(1.6 / 1.88)\end{array}$ & $\begin{array}{l}0.0977^{*} \\
0.0062^{\#}\end{array}$ & $\begin{array}{l}1.8 \\
(1.6 / 1.88)\end{array}$ & $\begin{array}{l}0.0202^{*} \\
0.0350^{\#}\end{array}$ & $\begin{array}{l}1.75 \\
(1.6 / 1.9)\end{array}$ & $\begin{array}{l}0.0419^{*} \\
0.0167^{\#}\end{array}$ & $\begin{array}{l}1.8 \\
(1.63 / 1.98)\end{array}$ & $\begin{array}{l}<0.0001^{*} \\
>0.9999^{\#}\end{array}$ \\
\hline Thrombin time, & $\begin{array}{l}17 \\
(17 / 18.8)\end{array}$ & $\begin{array}{l}27 \\
(22.5 / 30.8)\end{array}$ & $0.4338^{*}$ & $\begin{array}{l}14 \\
(14 / 15)\end{array}$ & $\begin{array}{r}0.0293^{*} \\
<0.0001^{\#}\end{array}$ & $\begin{array}{l}18 \\
(17 / 18.8)\end{array}$ & $\begin{array}{c}>0.9999^{*} \\
0.9336^{\#}\end{array}$ & $\begin{array}{l}12 \\
(12 / 12)\end{array}$ & $\begin{array}{r}0.0001^{*} \\
<0.0001^{\#}\end{array}$ & $\begin{array}{l}16 \\
(15 / 16)\end{array}$ & $\begin{array}{l}0.8270^{*} \\
0.0009^{\#}\end{array}$ \\
\hline
\end{tabular}

Data are presented as median (Q1/Q3). * Comparison to undiluted blood. ${ }^{*}$ Comparison to diluted blood.

fibrinogen substitution, $136 \mu \mathrm{L}(2.72 \mathrm{mg})$ of fibrinogen concentrate was added to $1,700 \mu \mathrm{L}$ of citrated blood $\left(1.6 \mathrm{mg} / \mathrm{mL}^{-1}\right.$ or $160 \mathrm{mg} /$ $\mathrm{dL}^{-1}$ ). This corresponds to a clinical dose of $100 \mathrm{mg} / \mathrm{kg}^{-1}$ (or $8 \mathrm{~g}$ per $80 \mathrm{~kg}$ body weight). All samples were stored at $37^{\circ} \mathrm{C}$ in a digital block heater (Dry Block Heater 3; IKA ${ }^{\circledR}$, Staufen, Germany) until ROTEM $^{\circledR}$ analyses were started, as described in previous studies.

\section{ROTEM $^{\circledR}$}

Thromboelastometric tests (EXTEM ${ }^{\circledR}$ and FIBTEM ${ }^{\circledR}$ ) were performed for each sample using ROTEM ${ }^{\circledR}$ delta analysers (TEM Innovations, Munich, Germany). Tests were performed according to manufacturer's instructions and terminated after $75 \mathrm{~min}$ $[4,10]$. For statistical analysis, we focussed on MCF measured by EXTEM $^{\circledR}\left(\right.$ MCF $\left._{\text {EXTEM }}\right)$ and FIBTEM $^{\circledR}($ MCF FIBTEM $)$ and CT (time from starting the test until a clot firmness amplitude of $2 \mathrm{~mm}$ has been achieved) by EXTEM $^{\circledR}\left(\mathrm{CT}_{\text {EXTEM }}\right)$. If the sample did not clot at all, CT was set to the maximum run time of $75 \mathrm{~min}$ $(4,500 \mathrm{~s})$

EXTEM $^{\circledR}$ uses tissue factor as activator and represents the extrinsic pathway of coagulation. FIBTEM $^{\circledR}$ contains cytochalasin D to block platelet function in addition to tissue factor. Accordingly, MCF $_{\text {FIBTEM }}{ }^{\circledR}$ is based on fibrin polymerization, only. ROTEM ${ }^{\circledR}$ analyses were performed on 3 ROTEM $^{\circledR}$ delta analysers, with different experimental conditions alternatively performed in a standardized fashion on all analysers. ROTEM ${ }^{\circledR}$ analyses were performed within $2 \mathrm{~h}$ after blood sampling.

Standard Laboratory Tests

Standard coagulation tests including international normalized ratio (INR; Thromborel S), thrombin time (TT; Berichrom Thrombinreagenz), and activated partial thromboplastin time (aPTT; Actin FSL; all from Siemens Healthcare GmbH, Erlangen, Germany) and blood counts were performed. Furthermore, fibrinogen plasma concentration as measured by the Clauss method was assessed (optical measurement, Multifibren U; Siemens Healthcare GmbH). All standard laboratory tests were performed by the Ludwig Maximilian University Institute for Laboratory Medicine, according to institutional standards. Tests were performed on BCS XP (Siemens Healthcare $\mathrm{GmbH}$ ).

\section{Statistical Analysis}

Data are presented as median with interquartile range unless otherwise indicated. Statistical analysis was performed using IBM $^{\circledR}$ SPSS $^{\circledR}$ Statistics version 24 (Armonk, NY, USA) and Graph Pad $^{\circledR}$ Prism 7 (La Jolla, CA, USA). Statistical differences between groups were analysed using the Friedmann test and Dunn's multiple comparison test. Correlation between $\mathrm{MCF}_{\text {FIBTEM }}$ and fibrinogen plasma concentration (Clauss method) was done by Spearman correlation. The alpha error was adjusted for multiple testing $(p=0.05 / n)$. The sample size was determined using $\mathrm{G}^{*}$ Power 3.1 (Dusseldorf, Germany) and an a priori power analysis ( $\alpha=0.05$; $\mathrm{dz}=0.8 ; 1-\beta=0.7)$ revealed 12 individuals per group.

\section{Results}

We assessed the performance of the 2 fibrinogen preparations fibryga ${ }^{\circledR}$ and Haemocomplettan ${ }^{\circledR} \mathrm{P}$ based on an in vitro dilutional coagulopathy model. All blood samples of the 12 healthy volunteers were taken into account.

\section{Volunteer Characteristics}

Detailed volunteer characteristics are displayed in Table 1 . None of them had known coagulation disorders or intake of anticoagulants.

\section{Sixty Percent Dilution with HES 6\% 130/0.4}

After dilution with 60\% HES all ROTEM parameters as well as standard laboratory parameters except for thrombin time deteriorated significantly (Table 2). Sub- 
Table 3. Dilution with gelatine

\begin{tabular}{|c|c|c|c|c|c|c|c|c|c|c|c|}
\hline Gelatine & $\begin{array}{l}\text { Undiluted } \\
\text { blood }\end{array}$ & $\begin{array}{l}60 \% \\
\text { dilution }\end{array}$ & $p$ & $\begin{array}{l}\text { fibryga } \\
\text { low dose }\end{array}$ & $p$ & $\begin{array}{l}\text { Haemocomplettan } \\
\text { low dose }\end{array}$ & $p$ & $\begin{array}{l}\text { fibryga } \\
\text { high dose }\end{array}$ & $p$ & $\begin{array}{l}\text { Haemocomplettan } \\
\text { high dose }\end{array}$ & $p$ \\
\hline $\mathrm{CT}_{\text {EXTEM }}, \mathrm{s}$ & $\begin{array}{l}59.5 \\
(54.75 / 62)\end{array}$ & $\begin{array}{l}95 \\
(86.75 / 102.8)\end{array}$ & $0.0499^{*}$ & $\begin{array}{l}63 \\
(60.3 / 71)\end{array}$ & $\begin{array}{c}>0.9999^{*} \\
0.6439^{*}\end{array}$ & $\begin{array}{l}51 \\
(47.5 / 54.5)\end{array}$ & $\begin{array}{r}0.3289^{*} \\
<0.0001^{\#}\end{array}$ & $\begin{array}{l}57.5 \\
(55,3 / 62.5)\end{array}$ & $\begin{array}{c}>0.9999^{*} \\
0.0244^{*}\end{array}$ & $\begin{array}{l}42.5 \\
(41 / 44.8)\end{array}$ & $\begin{array}{r}0.0033^{*} \\
<0.0001^{*}\end{array}$ \\
\hline $\mathrm{MCF}_{\mathrm{EXTEM}}, \mathrm{mm}$ & $\begin{array}{l}60 \\
(56.25 / 66)\end{array}$ & $\begin{array}{l}36.5 \\
(33.25 / 41.75)\end{array}$ & $<0.0001^{*}$ & $\begin{array}{l}45 \\
(43.3 / 49)\end{array}$ & $\begin{array}{l}0.0001^{*} \\
0.4338^{\#}\end{array}$ & $\begin{array}{l}45.5 \\
(42.25 / 49)\end{array}$ & $\begin{array}{c}<0.0001^{*} \\
0.9336^{\#}\end{array}$ & $\begin{array}{l}49 \\
(47.5 / 53)\end{array}$ & $\begin{array}{l}0.2129^{*} \\
0.0004^{\#}\end{array}$ & $\begin{array}{l}50 \\
(47.5 / 53.8)\end{array}$ & $\begin{array}{l}>0.9999^{*} \\
<0.0001^{*}\end{array}$ \\
\hline $\mathrm{MCF}_{\mathrm{FIBTEM}}, \mathrm{mm}$ & $\begin{array}{l}14 \\
(10.5 / 16)\end{array}$ & $\begin{array}{l}3 \\
(3 / 4)\end{array}$ & $<0.0001^{*}$ & $\begin{array}{l}8 \\
(6.5 / 9)\end{array}$ & $\begin{array}{c}0.0001^{*} \\
>0.9999^{\#}\end{array}$ & $\begin{array}{l}9 \\
(8 / 9)\end{array}$ & $\begin{array}{l}0.0167^{*} \\
0.1148^{\#}\end{array}$ & $\begin{array}{l}10 \\
(10 / 12)\end{array}$ & $\begin{array}{c}>0.9999^{*} \\
0.0006^{\#}\end{array}$ & $\begin{array}{l}14 \\
(13 / 15)\end{array}$ & $\begin{array}{l}>0.9999^{*} \\
<0.0001^{*}\end{array}$ \\
\hline $\begin{array}{l}\text { Fibrinogen } \\
\text { mg/asma, }\end{array}$ & $\begin{array}{l}257.5 \\
(220.5 / 339.8)\end{array}$ & $\begin{array}{l}76 \\
(66.25 / 95.75)\end{array}$ & $<0.0001^{*}$ & $\begin{array}{l}179.5 \\
(155.8 / 191.8)\end{array}$ & $\begin{array}{l}0.0202^{*} \\
0.2853^{\#}\end{array}$ & $\begin{array}{l}153.5 \\
(122.5 / 171.8)\end{array}$ & $\begin{array}{r}0.0017^{*} \\
>0.9999^{*}\end{array}$ & $\begin{array}{l}274 \\
(246 / 314)\end{array}$ & $\begin{array}{l}>0.9999^{*} \\
<0.0001^{\#}\end{array}$ & $\begin{array}{l}224.5 \\
(209.3 / 241.8)\end{array}$ & $\begin{array}{l}>0.9999^{*} \\
0.0004^{*}\end{array}$ \\
\hline aPTT, s & $\begin{array}{l}28 \\
(25.25 / 30)\end{array}$ & $\begin{array}{l}88.5 \\
(74.75 / 108)\end{array}$ & $<0.0001^{*}$ & $\begin{array}{l}81 \\
(70 / 97)\end{array}$ & $\begin{array}{l}0.0093^{*} \\
0.0829^{*}\end{array}$ & $\begin{array}{l}79.5 \\
(66.5 / 100.3)\end{array}$ & $\begin{array}{l}0.3289^{*} \\
0.0014^{*}\end{array}$ & $\begin{array}{l}81.5 \\
(68.3 /)\end{array}$ & $\begin{array}{l}0.0062^{*} \\
0.1148^{*}\end{array}$ & $\begin{array}{l}83.5 \\
(72 / 100.3)\end{array}$ & $\begin{array}{r}0.0001^{*} \\
>0.9999^{*}\end{array}$ \\
\hline INR & $\begin{array}{l}1 \\
(0.925 / 1)\end{array}$ & $\begin{array}{l}2.15 \\
(1.925 / 2.275)\end{array}$ & $<0.0001^{*}$ & $\begin{array}{l}1.75 \\
(1.6 / 1.9)\end{array}$ & $\begin{array}{l}0.1345^{*} \\
0.0009^{\#}\end{array}$ & $\begin{array}{l}1.8 \\
(1.7 / 2)\end{array}$ & $\begin{array}{l}0.0007^{*} \\
0.1572^{\#}\end{array}$ & $\begin{array}{l}1.75 \\
(1.63 / 1.9)\end{array}$ & $\begin{array}{l}0.0977^{*} \\
0.0014^{\#}\end{array}$ & $\begin{array}{l}1.85 \\
(1.63 / 1.9)\end{array}$ & $\begin{array}{l}0.0017^{*} \\
0.0829^{\#}\end{array}$ \\
\hline Thrombin time, s & $\begin{array}{l}17.5 \\
(17 / 18)\end{array}$ & $\begin{array}{l}35 \\
(30.5 / 38.75)\end{array}$ & $<0.0001^{*}$ & $\begin{array}{l}19.5 \\
(19 / 20.8)\end{array}$ & $\begin{array}{c}>0.9999^{*} \\
0.0011^{\#}\end{array}$ & $\begin{array}{l}27 \\
(25.25 / 29)\end{array}$ & $\begin{aligned} & 0.0007^{*} \\
&>0.9999^{*}\end{aligned}$ & $\begin{array}{l}16 \\
(16 / 16.8)\end{array}$ & $\begin{array}{l}>0.9999^{*} \\
<0.0001^{\#}\end{array}$ & $\begin{array}{l}24 \\
(24 / 25)\end{array}$ & $\begin{array}{l}0.0829^{*} \\
0.1148^{\#}\end{array}$ \\
\hline
\end{tabular}

Data are presented as median (Q1/Q3). * Comparison to undiluted blood. * Comparison to diluted blood.

Table 4. Dilution with albumin

\begin{tabular}{|c|c|c|c|c|c|c|c|c|c|c|c|}
\hline Albumin & $\begin{array}{l}\text { Undiluted } \\
\text { blood }\end{array}$ & $\begin{array}{l}60 \% \\
\text { dilution }\end{array}$ & $p$ & $\begin{array}{l}\text { fibryga } \\
\text { low dose }\end{array}$ & $p$ & $\begin{array}{l}\text { Haemocomplettan } \\
\text { low dose }\end{array}$ & $p$ & $\begin{array}{l}\text { fibryga } \\
\text { high dose }\end{array}$ & $p$ & $\begin{array}{l}\text { Haemocomplettan } \\
\text { high dose }\end{array}$ & $p$ \\
\hline $\mathrm{CT}_{\text {EXTEM }}, \mathrm{s}$ & $\begin{array}{l}57.5 \\
(54.25 / 61)\end{array}$ & $\begin{array}{l}89 \\
(81 / 94.75)\end{array}$ & $0.0022^{*}$ & $\begin{array}{l}69 \\
(66 / 75.5)\end{array}$ & $\begin{aligned} & 0.4338^{*} \\
> & 0.9999^{\#}\end{aligned}$ & $\begin{array}{l}56 \\
(53.25 / 57)\end{array}$ & $\begin{array}{l}>0.9999^{*} \\
<0.0001^{*}\end{array}$ & $\begin{array}{l}61 \\
(56.3 / 68,3)\end{array}$ & $\begin{array}{c}>0.9999^{*} \\
0.0499^{*}\end{array}$ & $\begin{array}{l}51 \\
(48.3 / 53)\end{array}$ & $\begin{array}{r}0.1148^{*} \\
<0.0001^{*}\end{array}$ \\
\hline $\mathrm{MCF}_{\text {EXTEM }}, \mathrm{mm}$ & $\begin{array}{l}60 \\
(56 / 66.5)\end{array}$ & $\begin{array}{l}41 \\
(37 / 41)\end{array}$ & $<0.0001^{*}$ & $\begin{array}{l}49 \\
(46.3 / 53.8)\end{array}$ & $\begin{array}{l}0.0004^{*} \\
0.2129^{*}\end{array}$ & $\begin{array}{l}48 \\
(46.25 / 52)\end{array}$ & $\begin{array}{l}<0.0001^{*} \\
>0.9999^{*}\end{array}$ & $\begin{array}{l}53 \\
(50.5 / 47)\end{array}$ & $\begin{array}{c}0.5660^{*} \\
<0.0001^{\#}\end{array}$ & $\begin{array}{l}52.5 \\
(50.5 / 57.75)\end{array}$ & $\begin{array}{r}0.7307^{*} \\
<0.0001^{*}\end{array}$ \\
\hline $\mathrm{MCF}_{\mathrm{FIBTEM}}, \mathrm{mm}$ & $\begin{array}{l}14 \\
(11 / 20)\end{array}$ & $\begin{array}{l}3.5 \\
(3 / 5)\end{array}$ & $<0.0001^{*}$ & $\begin{array}{l}8.5 \\
(8 / 10.75)\end{array}$ & $\begin{aligned} & 0.0051^{*} \\
> & 0.9999^{\#}\end{aligned}$ & $\begin{array}{l}10 \\
(9 / 11)\end{array}$ & $\begin{array}{l}0.1832^{*} \\
0.1572^{\#}\end{array}$ & $\begin{array}{l}14 \\
(13 / 15)\end{array}$ & $\begin{array}{l}>0.9999^{*} \\
<0.0001^{\#}\end{array}$ & $\begin{array}{l}18 \\
(16 / 19.75)\end{array}$ & $\begin{array}{l}>0.9999^{*} \\
<0.0001^{*}\end{array}$ \\
\hline Fibrinogen ${ }_{\text {Plasma }}, \mathrm{mg} / \mathrm{dL}$ & $\begin{array}{l}243.5 \\
(218.8 / 336.8)\end{array}$ & $\begin{array}{l}70.5 \\
(62.5 / 92.25)\end{array}$ & $<0.0001^{*}$ & $\begin{array}{l}163 \\
(150.3 / 187.3)\end{array}$ & $\begin{array}{l}0.0419^{*} \\
0.1148^{*}\end{array}$ & $\begin{array}{l}133.5 \\
(112.8 / 145)\end{array}$ & $\begin{array}{l}0.0003^{*} \\
>0.9999^{*}\end{array}$ & $\begin{array}{l}246 \\
(236.3 / 267)\end{array}$ & $\begin{array}{l}>0.9999^{*} \\
<0.0001^{\#}\end{array}$ & $\begin{array}{l}214.3 \\
(211 / 221)\end{array}$ & $\begin{array}{r}>0.9999^{*} \\
0.0004^{*}\end{array}$ \\
\hline aPTT, s & $\begin{array}{l}27.5 \\
(25.25 / 30)\end{array}$ & $\begin{array}{l}87.5 \\
(76.3 / 105.5)\end{array}$ & $<0.0001^{*}$ & $\begin{array}{l}83 \\
(66 / 99.75)\end{array}$ & $\begin{array}{l}0.0022^{*} \\
0.8270^{*}\end{array}$ & $\begin{array}{l}79 \\
(67 / 97.25)\end{array}$ & $\begin{array}{l}0.3289^{*} \\
0.0093^{\#}\end{array}$ & $\begin{array}{l}84 \\
(68.75 / 104)\end{array}$ & $\begin{array}{l}<0.0001^{*} \\
>0.9999^{*}\end{array}$ & $\begin{array}{l}81 \\
(64.5 / 102.3)\end{array}$ & $\begin{array}{l}0.0167^{*} \\
0.2129^{\#}\end{array}$ \\
\hline INR & $\begin{array}{l}1 \\
(1 / 1)\end{array}$ & $\begin{array}{l}2.2 \\
(2.03 / 2.48)\end{array}$ & $<0.0001^{*}$ & $\begin{array}{l}1.8 \\
(1.7 / 2.08)\end{array}$ & $\begin{array}{l}0.5660^{*} \\
0.0003^{*}\end{array}$ & $\begin{array}{l}1.95 \\
(1.83 / 2.1)\end{array}$ & $\begin{array}{l}0.0011^{*} \\
0.2468^{\#}\end{array}$ & $\begin{array}{l}1.95 \\
(1.8 / 2.1)\end{array}$ & $\begin{array}{l}0.0977^{*} \\
0.0041^{\#}\end{array}$ & $\begin{array}{l}2 \\
(1.8 / 2.2)\end{array}$ & $\begin{array}{l}0.0001^{*} \\
0.8270^{\#}\end{array}$ \\
\hline Thrombin time, s & $\begin{array}{l}17 \\
(17 / 18)\end{array}$ & $\begin{array}{l}30.5 \\
(27 / 33)\end{array}$ & $<0.0001^{*}$ & $\begin{array}{l}18 \\
(17 / 18)\end{array}$ & $\begin{array}{c}>0.9999^{*} \\
0.0002^{\#}\end{array}$ & $\begin{array}{l}24.5 \\
(24 / 25.75)\end{array}$ & $\begin{array}{r}0.0062^{*} \\
>0.9999^{*}\end{array}$ & $\begin{array}{l}15 \\
(15 / 15)\end{array}$ & $\begin{array}{c}0.9336^{*} \\
<0.0001^{\#}\end{array}$ & $\begin{array}{l}22.5 \\
(22 / 23)\end{array}$ & $\begin{array}{l}0.4338^{*} \\
0.1345^{\#}\end{array}$ \\
\hline
\end{tabular}

Data are presented as median (Q1/Q3). * Comparison to undiluted blood. . comparison to diluted blood.

stitution with low-dose fibryga ${ }^{\circledR}$ and low-dose Haemocomplettan ${ }^{\circledR}$ did not improve ROTEM parameters and standard laboratory parameters to values before dilution. Comparison of both preparations showed no significant difference (Table 2). Substitution with high-dose fibryga ${ }^{\circledR}$ and high-dose Haemocomplettan ${ }^{\circledR}$ improved ROTEM and standard laboratory parameters significantly compared to diluted blood. However, parameters did not reach undiluted blood values (Table 2). Comparison between both preparations showed no difference. The comparison between low-dose and high-dose fibryga ${ }^{\circledR}$ showed no differences except for the fibrinogen plasma concentration ( 219.5 vs. $\left.408 \mathrm{mg} / \mathrm{dL}^{-1} ; p=0.0017\right)$. Comparable results were observed with Haemocomplettan ${ }^{\circledR}(225.5$ vs. $\left.425.5 \mathrm{mg} / \mathrm{dL}^{-1} ; p=0.0011\right)$.

Of note, $\mathrm{MCF}_{\mathrm{FIBTEM}}$ and fibrinogen plasma concentration did not correlate $(r=0.2423 ; p=0.4697)$.

\section{Sixty Percent Dilution with Gelatine 4\%}

After dilution with $60 \%$ gelatine all ROTEM parameters as well as standard laboratory parameters deteriorated significantly (Table 3). Substitution with low-dose fibryga ${ }^{\circledR}$ and low-dose Haemocomplettan ${ }^{\circledR}$ improved the ROTEM parameters but not to values before dilution (Table 3). Comparison of both low-dose preparations showed no significant difference except for $\mathrm{CT}_{\text {EXTEM }}$ (63 vs. $51 \mathrm{~s} ; p=0.0202)$. Substitution with high-dose fibryga ${ }^{\circledR}$ and Haemocomplettan ${ }^{\circledR}$ corrected the ROTEM parameters to values of undiluted blood. Haemocomplettan ${ }^{\circledR}$ overcorrected the $\mathrm{CT}_{\text {EXTEM }}$ compared to undiluted blood (59.5 vs. $42.5 \mathrm{~s} ; p=0.0033$; Table 3). Standard laboratory tests were still significantly different to values of undiluted blood after high-dose substitution of both preparations except for thrombin time after fibryga ${ }^{\circledR}$ (17.5 vs. $16 \mathrm{~s}$; $p>0.999)$. Comparison between both high-dose preparations showed no differences except for the aforemen- 
Table 5. Dilution with balanced crystalloid

\begin{tabular}{|c|c|c|c|c|c|c|c|c|c|c|c|}
\hline $\begin{array}{l}\text { Balanced } \\
\text { crystalloid }\end{array}$ & Undiluted blood & $\begin{array}{l}60 \% \\
\text { dilution }\end{array}$ & $p$ & $\begin{array}{l}\text { fibryga } \\
\text { low dose }\end{array}$ & $p$ & $\begin{array}{l}\text { Haemocomplettan } \\
\text { low dose }\end{array}$ & $p$ & $\begin{array}{l}\text { fibryga } \\
\text { high dose }\end{array}$ & $p$ & $\begin{array}{l}\text { Haemocomplettan } \\
\text { high dose }\end{array}$ & $p$ \\
\hline $\mathrm{CT}_{\text {EXTEM }}, \mathrm{s}$ & $\begin{array}{l}58.5 \\
(56.3 / 63.8)\end{array}$ & $\begin{array}{l}65 \\
(60.3 / 69.8)\end{array}$ & $>0.9999^{*}$ & $\begin{array}{l}53.5 \\
(53 / 57.8 /)\end{array}$ & $\begin{array}{c}>0.9999^{*} \\
0.1345^{\#}\end{array}$ & $\begin{array}{l}45 \\
(43 / 47)\end{array}$ & $\begin{array}{l}<0.0001^{*} \\
<0.0001^{\#}\end{array}$ & $\begin{array}{l}52.5 \\
(48.5 / 56.8)\end{array}$ & $\begin{array}{l}0.3289^{*} \\
0.0167^{*}\end{array}$ & $\begin{array}{l}45.5 \\
(41 / 46.75)\end{array}$ & $\begin{array}{l}<0.0001^{*} \\
<0.0001^{*}\end{array}$ \\
\hline $\mathrm{MCF}_{\mathrm{EXTEM}}, \mathrm{mm}$ & $\begin{array}{l}60.5 \\
(56 / 64.8)\end{array}$ & $\begin{array}{l}41 \\
(37.3 / 45.8)\end{array}$ & $<0.0001^{*}$ & $\begin{array}{l}50 \\
(48 / 53)\end{array}$ & $\begin{array}{l}0.0001^{*} \\
0.5660^{*}\end{array}$ & $\begin{array}{l}50 \\
(46.5 / 53.8)\end{array}$ & $\begin{array}{c}<0.0001^{*} \\
0.7307^{\#}\end{array}$ & $\begin{array}{l}54.5 \\
(51.5 / 57.75)\end{array}$ & $\begin{array}{l}0.2468^{*} \\
0.0004^{*}\end{array}$ & $\begin{array}{l}56 \\
(52.25 / 59.5)\end{array}$ & $\begin{array}{l}>0.9999^{*} \\
<0.0001^{*}\end{array}$ \\
\hline $\mathrm{MCF}_{\mathrm{FIBTEM}}, \mathrm{mm}$ & $\begin{array}{l}14.5 \\
(10.3 / 16)\end{array}$ & $\begin{array}{l}5.5 \\
(4 / 6)\end{array}$ & $0.4338^{*}$ & $\begin{array}{l}13 \\
(11.3 / 14.8)\end{array}$ & $\begin{array}{c}>0.9999^{*} \\
0.9336^{\#}\end{array}$ & $\begin{array}{l}17 \\
(14.3 / 18.8)\end{array}$ & $\begin{array}{l}0.7307^{*} \\
0.0007^{*}\end{array}$ & $\begin{array}{l}19.5 \\
(18 / 21.75)\end{array}$ & $\begin{array}{r}0.0350^{*} \\
<0.0001^{\#}\end{array}$ & $\begin{array}{l}25.5 \\
(23.25 / 29.25)\end{array}$ & $\begin{array}{r}0.0001^{*} \\
<0.0001^{*}\end{array}$ \\
\hline Fibrinogen ${ }_{\text {Plasma }}, \mathrm{mg} / \mathrm{dL}$ & $\begin{array}{l}270.5 \\
(212 / 329.5)\end{array}$ & $\begin{array}{l}62 \\
(48.5 / 81.3)\end{array}$ & $<0.0001^{*}$ & $\begin{array}{l}164 \\
(144 / 182.3)\end{array}$ & $\begin{array}{l}0.0093^{*} \\
0.1148^{\#}\end{array}$ & $\begin{array}{l}112 \\
(109 / 135.3)\end{array}$ & $\begin{array}{l}<0.0001^{*} \\
>0.9999^{\#}\end{array}$ & $\begin{array}{l}246 \\
(235.8 / 267.8)\end{array}$ & $\begin{array}{l}>0.9999^{*} \\
<0.0001^{\#}\end{array}$ & $\begin{array}{l}216 \\
(198.3 / 228.8)\end{array}$ & $\begin{array}{l}0.4962^{*} \\
0.0011^{\#}\end{array}$ \\
\hline aPTT, s & $\begin{array}{l}28 \\
(25.3 / 31.5)\end{array}$ & $\begin{array}{l}79.5 \\
(71.5 / 94)\end{array}$ & $<0.0001^{*}$ & $\begin{array}{l}77 \\
(60.8 / 94.3)\end{array}$ & $\begin{array}{r}0.0027^{*} \\
>0.9999^{\#}\end{array}$ & $\begin{array}{l}71 \\
(61 / 93)\end{array}$ & $\begin{array}{l}0.0977^{*} \\
0.1148^{\#}\end{array}$ & $\begin{array}{l}74.5 \\
(60.8 / 98.8)\end{array}$ & $\begin{array}{c}0.0001^{*} \\
>0.9999^{\#}\end{array}$ & $\begin{array}{l}73.5 \\
(63.5(93.5)\end{array}$ & $\begin{array}{l}0.0062^{*} \\
0.9336^{\#}\end{array}$ \\
\hline INR & $\begin{array}{l}1 \\
(1 / 1)\end{array}$ & $\begin{array}{l}2.1 \\
(1.93 / 2.28)\end{array}$ & $<0.0001^{*}$ & $\begin{array}{l}1.75 \\
(1.7 / 1.9)\end{array}$ & $\begin{array}{l}0.3783^{*} \\
0.0004^{*}\end{array}$ & $\begin{array}{l}1.85 \\
(1.73 / 2)\end{array}$ & $\begin{array}{l}0.0006^{*} \\
0.3289^{\#}\end{array}$ & $\begin{array}{l}1.75 \\
(1.63 / 1.9)\end{array}$ & $\begin{array}{l}0.3783^{*} \\
0.0004^{*}\end{array}$ & $\begin{array}{l}1.9 \\
(1.73 / 2)\end{array}$ & $\begin{array}{l}<0.0001^{*} \\
>0.9999^{*}\end{array}$ \\
\hline Thrombin time, s & $\begin{array}{l}17.5 \\
(17 / 18)\end{array}$ & $\begin{array}{l}28 \\
(25.3 / 30.8)\end{array}$ & $0.0011^{*}$ & $\begin{array}{l}17 \\
(16 / 17)\end{array}$ & $\begin{array}{l}>0.9999^{*} \\
<0.0001^{\#}\end{array}$ & $\begin{array}{l}23.5 \\
(23 / 24)\end{array}$ & $\begin{array}{c}0.0499^{*} \\
>0.9999^{\#}\end{array}$ & $\begin{array}{l}14 \\
(14 / 14)\end{array}$ & $\begin{array}{c}0.1832^{*} \\
<0.0001^{\#}\end{array}$ & $\begin{array}{l}22 \\
(21 / 22.8)\end{array}$ & $\begin{array}{r}>0.9999^{*} \\
0.2129^{*}\end{array}$ \\
\hline
\end{tabular}

Data are presented as median (Q1/Q3). * Comparison to undiluted blood. ${ }^{*}$ Comparison to diluted blood.

tioned $\mathrm{CT}_{\text {EXTEM }}(57.5$ vs. $42.5 \mathrm{~s} ; p=0.0076)$ and TT (16 vs. $24 \mathrm{~s} ; p=0.0017$ ). Comparing low-dose and highdose fibryga ${ }^{\circledR}$ showed no differences except for fibrinogen plasma concentrations. Comparing low-dose and high-dose Haemocomplettan ${ }^{\circledR}$ showed differences in $\mathrm{MCF}_{\text {FIBTEM }}\left(9\right.$ vs. $14 \mathrm{~mm} ; p=0.0244$ ) and $\mathrm{MCF}_{\text {EXTEM }}$ ( 45.5 vs. $50 \mathrm{~mm} ; p=0.0167$ ).

A correlation of $\mathrm{MCF}_{\text {FIBTEM }}$ and fibrinogen plasma concentration was observed; however, it was not statistically significant $(r=0.5682 ; p=0.0520)$.

\section{Sixty Percent Dilution with Human Albumin 5\%}

After dilution with 60\% human albumin all ROTEM parameters as well as standard laboratory parameters deteriorated significantly (Table 4). Substitution with lowdose fibryga ${ }^{\circledR}$ and Haemocomplettan ${ }^{\circledR}$ improved all of the parameters, but most were lower than before dilution. In detail, Haemocomplettan ${ }^{\circledR}$ only improved $\mathrm{CT}_{\text {EXTEM }}$

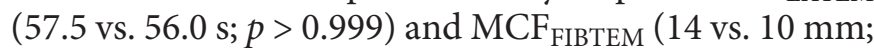
$p=0.1832$ ) to values before dilution (Table 4), and fibryga ${ }^{\circledR}$ corrected TT to values of undiluted blood (17 vs. $18 \mathrm{~s} ; p>0.999)$. Thus, comparing low-dose fibryga ${ }^{\circledR}$ and Haemocomplettan ${ }^{\circledR}$ showed differences in $\mathrm{CT}_{\text {EXTEM }}(69$ vs. $56 \mathrm{~s} ; p=0.0114$ ) and TT (18 vs. $24.5 \mathrm{~s} ; p=0.0202$ ). Comparison of high-dose fibryga ${ }^{\circledR}$ and Haemocomplet$\tan ^{\circledR}$ corrected all parameters to values of undiluted blood except for INR (Table 4), and comparison showed differences between both preparations for $\mathrm{CT}_{\text {EXTEM }}$ (61 vs. $51 \mathrm{~s} ; p=0.0062)$ and TT (15 vs. $22.5 \mathrm{~s} ; p=0.0011)$.

Comparison of low-dose and high-dose fibryga ${ }^{\circledR}$ presented significant differences for MCF $_{\text {FIBTEM }}$ (8.5 vs. 14 $\mathrm{mm} ; p=0.0244)$ and fibrinogen plasma concentrations ( $163 \mathrm{vs.} 246 \mathrm{mg} / \mathrm{dL}^{-1} ; p=0.0093$ ). Comparing low-dose and high-dose Haemocomplettan ${ }^{\circledR}$ showed the same results but also a difference in $\mathrm{MCF}_{\text {EXTEM }}$ (48 vs. $52.5 \mathrm{~mm} ; p=0.0244$ ).
After dilution with human albumin, $\mathrm{MCF}_{\mathrm{FIBTEM}}$ and fibrinogen plasma concentration showed a strong correlation $(r=0.87 ; p=0.0005)$.

Sixty Percent Dilution with Balanced Ringer's Acetate

After dilution with $60 \%$ crystalloid solution only

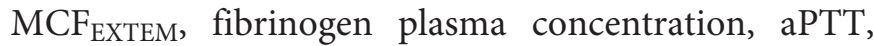
INR, and TT deteriorated significantly (Table 5). Substitution of low-dose fibryga ${ }^{\circledR}$ already corrected $\mathrm{CT}_{\text {EXTEM}}$, $\mathrm{MCF}_{\text {FIBTEM, }}$ and INR to values of undiluted blood (Table 5). Haemocomplettan ${ }^{\circledR}$ corrected MCF FIBTEM and aPTT and overcorrected $\mathrm{CT}_{\text {EXTEM. }}$. Comparison of both preparations showed significant differences in $\mathrm{CT}_{\text {EXTEM }}(53.5$ vs. $45.5 \mathrm{~s} ; p=0.035)$ and TT (17 vs. $23.5 \mathrm{~s} ; p=0.0014$ ). Substitution of high-dose fibryga ${ }^{\circledR}$ and Haemocomplet$\tan { }^{\circledR}$ corrected CT $\mathrm{EXXEM}_{\text {(again outperformed correction }}$ with Haemocomplettan $\left.{ }^{\circledR}\right), \mathrm{MCF}_{\text {EXTEM }}$, fibrinogen plasma concentration, and TT (Table 5). Comparison of both preparations showed differences in TT (14 vs. $22 \mathrm{~s} ; p=$ 0.0009). Comparing low-dose and high-dose fibryga ${ }^{\circledR}$ showed differences for $\mathrm{MCF}_{\text {FIBTEM }}(13$ vs. $19.5 \mathrm{~mm} ; p=$ 0.0114 ) and fibrinogen plasma concentrations (164 vs. $\left.246 \mathrm{mg} / \mathrm{dL}^{-1} ; p=0.0202\right)$. Comparison of the 2 Haemocomplettan ${ }^{\circledR}$ preparations showed differences in

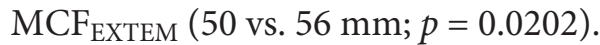

MCF $_{\text {FIBTEM }}$ and fibrinogen plasma concentration showed a strong correlation $(r=0.89667 ; p=0.0002)$.

\section{Discussion/Conclusion}

In this study we show, that the new fibrinogen concentrate fibryga ${ }^{\circledR}$ and the established product Haemocomplettan ${ }^{\circledR}$ had comparable effects in our in vitro model of dilutional coagulopathy. However, minor differences in 
thrombin time and $\mathrm{CT}_{\text {EXTEM }}$ between both preparations were seen. fibryga ${ }^{\circledR}$ had a stronger impact on thrombin time compared to Haemocomplettan ${ }^{\circledR}$, that is, due to factor XIII-related improvement in fibrin polymerization. Additionally, Haemocomplettan ${ }^{\circledR}$ had a more pronounced effect on shortening of $\mathrm{CT}_{\text {EXTEM }}$ which might be due to increased factor VIII concentration compared to fibryga ${ }^{\circledR}$.

Coagulopathy in severely injured patients with high blood loss is characterized by dilution, consumption of coagulation factors, and critically low fibrinogen plasma concentrations. As fibrinogen is often one of the first coagulation factors to reach critically low plasma concentrations, early administration of fibrinogen and tranexamic acid in cases of heavy trauma and blood loss is being proposed $[11,12]$. Thus, the European guidelines recommend fibrinogen administration if plasma concentrations fall below concentrations of $150-200 \mathrm{mg} / \mathrm{dL}^{-1}$ [13] Usually between 50 and $100 \mathrm{mg} / \mathrm{kg}^{-1}$ body weight of fibrinogen are administered in severe bleeding and relevant blood loss $[14,15]$. Thus, we analysed both 50 and $100 \mathrm{mg} / \mathrm{kg}^{-1}$ fibrinogen administration to simulate lowdose and high-dose fibrinogen substitution of both preparations and its performance. We used 4 common diluents to investigate the performance of the 2 preparations. The influence and impact on coagulation of those diluents are well known and therefore eligible for our model $[9,16-21]$.

Our results with the crystalloid model, showing similar impact of fibryga ${ }^{\circledR}$ and Haemocomplettan ${ }^{\circledR}$, are in accordance with a previously published analysis of Haas et al. [18]. This group also compared the performance of the 2 fibrinogen preparations as it was done in this study. In contrast to our study this group used a model with crystalloid only. Therefore, this is the first study to analyse the performance of fibryga ${ }^{\circledR}$ and Haemocomplettan ${ }^{\circledR}$ under different conditions. Haas et al. [18] used different lots and compared the amount of fibrinogen to calculate a factor to adjust the samples. The group could demonstrate a distinctive increase of $\mathrm{MCF}_{\text {EXTEM }}$ after substitution with fibryga ${ }^{\circledR}$. This effect could not be seen with our data. Besides, they did not evaluate the dynamics of clot formation as we did with $\mathrm{CT}_{\text {EXTEM }}$ and did not use standard laboratory tests. In a prior study comparing both preparations in congenital fibrinogen deficiency, no differences in $\mathrm{MCF}_{\text {FIBTEM }}$ and no differences in complication rate were found [22].

An increase in $\mathrm{MCF}_{\text {EXTEM }}$ and $\mathrm{MCF}_{\mathrm{FIBTEM}}$ after fibrinogen replacement was seen in the presence of all diluents, which is in line with the results of prior studies evaluating the effect of fibrinogen substitution on these parameters $[4,6]$. They postulate that the sole administration of fibrinogen particularly influences clot stability and less the dynamics of clot formation. Only high-dose fibrinogen substitution slightly influenced clot formation dynamics [6]. In the present study, the dynamics of both clot formation and clot stability were influenced. In particular, Haemocomplettan ${ }^{\circledR}$ had an impact on the dynamics of clot formation, as seen by improvement of the $\mathrm{CT}_{\text {EXTEM. }}$ Effects on clot dynamics is possibly caused by slight differences of co-factors (e.g., FVIII) within the preparation of the fibrinogen product.

As expected, HES had a profound effect on ROTEM ${ }^{\circledR}$ parameters, which were only partly restored even by high-dose fibryga ${ }^{\circledR}$ and Haemocomplettan ${ }^{\circledR}$ administration $[9,18]$. This confirms the data of Schlimp et al. [8]. Fibrinogen plasma concentrations assessed using the Clauss method (functional assay with optical measurement of the clot formation time in the laboratory) differed from ROTEM ${ }^{\circledR}$ results, with no correlation between $\mathrm{MCF}_{\text {FIBTEM }}$ and fibrinogen plasma concentration which is a well-known effect. It can be explained by methodological issues [23]. Fibrinogen plasma concentration using the Clauss method is assessed photometrically and can be disturbed by HES [23-26]. Thus, fibrinogen plasma concentration is overestimated, and low fibrinogen plasma concentrations can be mimicked when HES is present in the probe, which leads to bad correlations of those optical tests with other tests [23-26]. The impact of the other diluents was in accordance with prior publications and showed deteriorated coagulation parameters as expected $[9,17,18,21]$.

The effects seen in ROTEM ${ }^{\circledR}$ analyses were compared (not correlated) to the changes in standard laboratory coagulation tests. INR and aPTT did not change significantly after fibrinogen substitution, whereas TT increased especially after administration of fibryga ${ }^{\circledR}$. TT was significantly shorter in contrast to supplementation with Haemocomplettan ${ }^{\circledR}$. The reason for this effect is still not completely clear. One explanation can be the effect due to FXIII-related improvement in fibrin polymerization as described in the manufacturer's information. Some of our observed differences might be explained by differences in the exact dosage of fibrinogen despite the labelled and standardized dosage of the factor concentrates: using fibrinogen concentrates, clinicians might think that $1 \mathrm{~g}$ of fibrinogen concentrate contains exactly $1 \mathrm{~g}$ of fibrinogen. Due to rules and regulations, quality must ensure that a fibrinogen concentrate contains at least the indicated amount of fibrinogen. However, some range - both below and above the target value - is allowed by the "National Federal Institute for Drugs and Medical Devices" and other public health authorities. This explains differences in the actual amount of fibrinogen in a specific product. Comparing fibryga ${ }^{\circledR}$ and Haemocomplettan ${ }^{\circledR} \mathrm{P}$, it turns out that in the specific lot used for our study, fibryga ${ }^{\circledR}$ contained $27.3 \%$ less fibrinogen than Haemocomplet$\tan ^{\circledR} \mathrm{P}$ measured by the Clauss method (information is 
part of the quality data provided by the manufacturer). Immunological measurement of fibrinogen showed only $4.3 \%$ difference between the preparations. Both lots were in the allowed range. This can serve as an explanation for the few but not significant differences in $\mathrm{MCF}_{\mathrm{EXTEM}}$ and $\mathrm{MCF}_{\text {FIBTEM }}$ and the significant improvement of $\mathrm{CT}_{\text {EXTEM }}$ after Haemocomplettan ${ }^{\circledR}$. The observed slightly higher impact of fibryga ${ }^{\circledR}$ when compared to Haemocomplet$\tan ^{\circledR}$ on thrombin time, despite no differences in other standard laboratory values, nonetheless remains difficult to understand. However, it should be taken into account that despite the similar active compound of fibrinogen the preparations may vary slightly in composition due to differences in production. Therefore, a specific effect of one distinct constituent potentially interfering with single laboratory tests cannot be excluded completely.

Our study has limitations. We simulated dilutional coagulopathy in vitro and the results are based on standardized conditions, which would vary in vivo, for example, in the operating room. In detail, sample temperature, citrate concentrations, time until test initiation, and so on, which influence results were all standardized throughout this study and, thus, did not mimic the in vivo situation. The influence of those cannot be seen with standardized coagulation tests (standard laboratory as well as thromboelastometry) because both are performed, for example, at $37^{\circ} \mathrm{C}$ and following recalcification and can differ, therefore, from a real situation. Furthermore, dilutional coagulopathy in vivo is a complex setting and influenced by additional factors like endothelial integrity, glycocalyx shedding, endogenous heparinoids, temperature, platelet function, and so on, none of which has been analysed in our study [27-30]. The standard laboratory tests used do not reflect an in vivo clotting situation, that is, fibrinogen analysed by the Clauss method is influenced by colloids. Thus, results might not be completely comparable to the thromboelastometric assays used. This is of particular interest, as thromboelastometric assays better represent whole blood coagulation.

Dilution by a single preparation is not common and does not reflect the real situation in the operating room. However, it can be used as a model to evaluate the performance of different fibrinogen preparations with standardized conditions.

In conclusion, both fibryga ${ }^{\circledR}$ and Haemocomplettan ${ }^{\circledR}$ $\mathrm{P}$, as commercially available fibrinogen concentrates, had the same performance in an in vitro model of dilutional coagulopathy. As expected, higher doses of $100 \mathrm{mg} / \mathrm{kg}^{-1}$ of both fibrinogen concentrates proved to be more effective than $50 \mathrm{mg} / \mathrm{kg}^{-1}$ in this model, which should be evaluated in clinical, randomized studies. Minor differences between the 2 concentrates were observed in some of the ROTEM $^{\circledR}$ variables depending on the different diluents. Minor differences could also be observed in the standard laboratory tests with a slightly higher impact of unknown significance of fibryga ${ }^{\circledR}$ on thrombin time compared to Haemocomplettan ${ }^{\circledR}$.

\section{Acknowledgements}

The authors would like to express thanks for the kind support of the staff of Professor Alexander Chouker and his group from the research laboratory on stress and immunity, Department of Anaesthesiology, University of Munich, Germany. Furthermore, we thank Professor Lesca Holdt and Mrs. Pauli from the department of "Laboratoriumsmedizin" for performing standard laboratory tests.

\section{Statement of Ethics}

This in vitro study was approved by the Ludwig Maximilian University Ethics Committee (No. 17-525) and performed in accordance with the Declaration of Helsinki. Healthy volunteers were recruited by bulletins at our institution and enrolled after written informed consent.

\section{Disclosure Statement}

S.T.S. received lecture fees from CSL Behring. The other authors have no conflicts of interest to declare.

\section{Funding Sources}

This work was supported by departmental sources and in part by a research grant to Dr. Schäfer from Octapharma, Langenfeld, Germany. The funding sponsors (Octapharma) had no role in the design of the study; in the collection, analyses, or interpretation of data; in the writing of the manuscript; or in the decision to publish the results.

\section{Author Contributions}

P.G.: study design, data collection, statistical analysis, and manuscript writing. T.W.: data collection, statistical analysis, and manuscript writing. T.K., P.M., M.M., and A.B.: statistical analysis and manuscript writing; K.G.: statistical analysis, interpretation of data and manuscript writing; M.R.: interpretation of data and manuscript writing; S.T.S.: study design, data collection, statistical analysis, interpretation of data, and manuscript writing.

References

1 Floccard B, Rugeri L, Faure A, Saint Denis M, Boyle EM, Peguet O, et al. Early coagulopathy in trauma patients: an on-scene and hospital admission study. Injury. 2012 Jan;43(1):2632.

2 Rourke C, Curry N, Khan S, Taylor R, Raza I, Davenport R, et al. Fibrinogen levels during trauma hemorrhage, response to replacement therapy, and association with patient outcomes. J Thromb Haemost. 2012 Jul;10(7): 1342-51. 
3 Hayakawa M, Gando S, Ono Y, Wada T, Yanagida Y, Sawamura A. Fibrinogen level deteriorates before other routine coagulation parameters and massive transfusion in the early phase of severe trauma: a retrospective observational study. Semin Thromb Hemost. 2015 Feb;41(1):35-42.

4 Schmidt DE, Halmin M, Wikman A, Östlund A, Ågren A. Relative effects of plasma, fibrinogen concentrate, and factor XIII on ROTEM coagulation profiles in an in vitro model of massive transfusion in trauma. Scand J Clin Lab Invest. 2017 Oct;77(6):397-405.

5 Ponschab M, Voelckel W, Pavelka M, Schlimp CJ, Schöchl H. Effect of coagulation factor concentrate administration on ROTEM $^{\circledR}$ parameters in major trauma. Scand J Trauma Resusc Emerg Med. 2015 Oct;23(1):84.

6 Schäfer N, Driessen A, Bauerfeind U, Fröhlich $M$, Ofir J, Stürmer EK, et al. In vitro effects of different sources of fibrinogen supplementation on clot initiation and stability in a model of dilutional coagulopathy. Transfus Med. 2016 Oct;26(5):373-80.

7 Haas T, Fries D, Velik-Salchner C, Reif C, Klingler A, Innerhofer P. The in vitro effects of fibrinogen concentrate, factor XIII and fresh frozen plasma on impaired clot formation after 60\% dilution. Anesth Analg. 2008 May;106(5):1360-5, table of contents.

8 Schlimp CJ, Cadamuro J, Solomon C, Redl H, Schöchl H. The effect of fibrinogen concentrate and factor XIII on thromboelastometry in $33 \%$ diluted blood with albumin, gelatine, hydroxyethyl starch or saline in vitro. Blood Transfus. 2013 Oct;11(4):510-7.

9 De Lorenzo C, Calatzis A, Welsch U, Heindl B. Fibrinogen concentrate reverses dilutional coagulopathy induced in vitro by saline but not by hydroxyethyl starch 6\%. Anesth Analg. 2006 Apr;102(4):1194-200.

10 Whiting D, DiNardo JA. TEG and ROTEM: technology and clinical applications. Am J Hematol. 2014 Feb;89(2):228-32.

11 Franchini M, Lippi G. Fibrinogen replacement therapy: a critical review of the literature. Blood Transfus. 2012 Jan;10(1):23-7.
12 Schlimp CJ, Schöchl H. The role of fibrinogen in trauma-induced coagulopathy. Hamostaseologie. 2014;34(1):29-39.

13 Spahn DR, Bouillon B, Cerny V, Duranteau J, Filipescu D, Hunt BJ, et al. The European guideline on management of major bleeding and coagulopathy following trauma: fifth edition. Crit Care. 2019 Mar;23(1):98.

14 Gaarder C, Naess PA, Frischknecht Christensen E, Hakala P, Handolin L, Heier HE, et al. Scandinavian Guidelines-"The massively bleeding patient". Scand J Surg. 2008;97(1): 15-36.

15 Spahn DR, Rossaint R. Coagulopathy and blood component transfusion in trauma. Br J Anaesth. 2005 Aug;95(2):130-9.

16 Engström M, Reinstrup P, Schött U. An in vitro evaluation of standard rotational thromboelastography in monitoring of effects of recombinant factor VIIa on coagulopathy induced by hydroxy ethyl starch. BMC Blood Disord. 2005 Feb;5(1):3.

17 Mauch J, Madjdpour C, Kutter AP, Spielmann N, Bettschart-Wolfensberger R, Weiss $\mathrm{M}$, et al. Effect of rapid fluid resuscitation using crystalloids or colloids on hemostasis in piglets. Paediatr Anaesth. 2013 Mar;23(3): 258-64.

18 Haas T, Preinreich A, Oswald E, Pajk W, Berger J, Kuehbacher G, et al. Effects of albumin 5\% and artificial colloids on clot formation in small infants. Anaesthesia. 2007 Oct; 62(10):1000-7.

19 Sigurjonsson J, Hedman D, Bansch P, Schött U. Comparison of dextran and albumin on blood coagulation in patients undergoing major gynaecological surgery. Perioper Med (Lond). 2018 Sep;7(1):21.

20 Moeller C, Fleischmann C, Thomas-Rueddel D, Vlasakov V, Rochwerg B, Theurer P, et al. How safe is gelatin? A systematic review and meta-analysis of gelatin-containing plasma expanders vs crystalloids and albumin. J Crit Care. 2016 Oct;35:75-83.

21 Rasmussen KC, Secher NH, Pedersen T. Effect of perioperative crystalloid or colloid fluid therapy on hemorrhage, coagulation competence, and outcome: A systematic review and stratified meta-analysis. Medicine (Baltimore). 2016 Aug;95(31):e4498.
22 Ross C, Rangarajan S, Karimi M, Toogeh G, Apte S, Lissitchkov T, et al. Pharmacokinetics, clot strength and safety of a new fibrinogen concentrate: randomized comparison with active control in congenital fibrinogen deficiency. J Thromb Haemost. 2018 Feb;16(2): 253-61.

23 Adam S, Karger R, Kretschmer V. Photo-optical methods can lead to clinically relevant overestimation of fibrinogen concentration in plasma diluted with hydroxyethyl starch. Clin Appl Thromb Hemost. 2010 Aug;16(4): 461-71.

24 Hiippala ST. Dextran and hydroxyethyl starch interfere with fibrinogen assays. Blood Coagul Fibrinolysis. 1995 Dec;6(8):743-6.

25 Fenger-Eriksen C, Moore GW, Rangarajan S, Ingerslev J, Sørensen B. Fibrinogen estimates are influenced by methods of measurement and hemodilution with colloid plasma expanders. Transfusion. $2010 \mathrm{Dec} ; 50$ (12):25716.

26 Adam S, Karger R, Kretschmer V. Influence of different hydroxyethyl starch (HES) formulations on fibrinogen measurement in HES-diluted plasma. Clin Appl Thromb Hemost. 2010 Aug;16(4):454-60.

27 Chappell D, Bruegger D, Potzel J, Jacob M, Brettner F, Vogeser M, et al. Hypervolemia increases release of atrial natriuretic peptide and shedding of the endothelial glycocalyx. Crit Care. 2014 Oct;18(5):538.

28 Chappell D, Jacob M. Role of the glycocalyx in fluid management: small things matter. Best Pract Res Clin Anaesthesiol. 2014 Sep; 28(3):227-34

29 Cap A, Hunt BJ. The pathogenesis of traumatic coagulopathy. Anaesthesia. 2015 Jan;70 Suppl 1:96-101, e32-4.

30 Lier H, Krep H, Schroeder S, Stuber F. Preconditions of hemostasis in trauma: a review. The influence of acidosis, hypocalcemia, anemia, and hypothermia on functional hemostasis in trauma. J Trauma. 2008 Oct;65(4): 951-60. 